\title{
Advanced Helical Plasma Research towards a Steady-State Fusion Reactor by Deuterium Experiments in Large Helical Device
}

\author{
Yasuhiko Takeiri ${ }^{1,2}$ (D) \\ 1 National Institute for Fusion Science, National Institutes of Natural Sciences, 322-6 Oroshi, \\ Toki 509-5292, Japan; takeiri@nifs.ac.jp; Tel.: +81-572-58-2008 \\ 2 SOKENDAI (The Graduate University for Advanced Studies), 322-6 Oroshi, Toki 509-5292, Japan
}

Received: 3 October 2018; Accepted: 4 December 2018; Published: 8 December 2018

\begin{abstract}
The Large Helical Device (LHD) is one of the world's largest superconducting helical system fusion-experiment devices. Since the start of experiments in 1998, it has expanded its parameter regime. It has also demonstrated world-leading steady-state operation. Based on this progress, the LHD has moved on to the advanced research phase, that is, deuterium experiment, which started in March 2017. During the first deuterium experiment campaign, an ion temperature of $10 \mathrm{keV}$ was achieved. This was a milestone in helical systems research: demonstrating one of the conditions for fusion. All of this progress and increased understanding have provided the basis for designing an LHD-type steady-state helical fusion reactor. Moreover, LHD plasmas have been utilized not only for fusion research, but also for diagnostics development and applications in wide-ranging plasma research. A few examples of such contributions of LHD plasmas (spectroscopic study and the development of a new type of interferometer) are introduced in this paper.
\end{abstract}

Keywords: Large Helical Device (LHD); deuterium experiment; ion temperature of $10 \mathrm{keV}$; plasma research; spectroscopic study; dispersion interferometer

\section{Introduction}

The Large Helical Device (LHD) [1] is one of the world's largest magnetically-confined fusion-experiment devices and is categorized as a helical system. Experiments started in March 1998, and the LHD has taken part in pioneering research in the worldwide fusion research community since then. The LHD has the critical advantage and engineering capability of steady-state operation. It has played a complementary and alternative role to the tokamak approach. The main goals of the LHD are to establish a scientific basis for a steady-state helical fusion reactor and to promote academic study for a comprehensive physics-based understanding of toroidal plasmas.

It is worth noting that helical fusion research has been performed worldwide, as shown in Figure 1 [2]. Another large-scale superconducting device, Wendelstein 7-X, started operation in 2015 [3]. Costa Rica [4] and China have also commenced helical fusion research. In July 2017, the National Institute for Fusion Science (NIFS) agreed with Southwest Jiaotong University to collaboratively construct, and then conduct fusion research using, the Chinese First Quasi-Axisymmetric Stellarator (CFQS) [5]. The start of its experiments is envisaged for 2021.

This paper is organized as follows. In Section 2, one significant achievement (i.e., an ion temperature record) and a few other wide-ranging physics results obtained in the LHD's first deuterium campaign are briefly described. Note that most of these results are before publication, and details will be given in future individual publications. Section 3 is devoted to introducing the design activity of a steady-state helical fusion reactor-the so-called FFHR. Section 4 emphasizes LHD 
as a platform for wide-ranging plasma research by describing two examples of its use within such research. These examples are the LHD's use in spectroscopic study, and the development of a new type of interferometer that is applicable to atmospheric pressure plasmas.

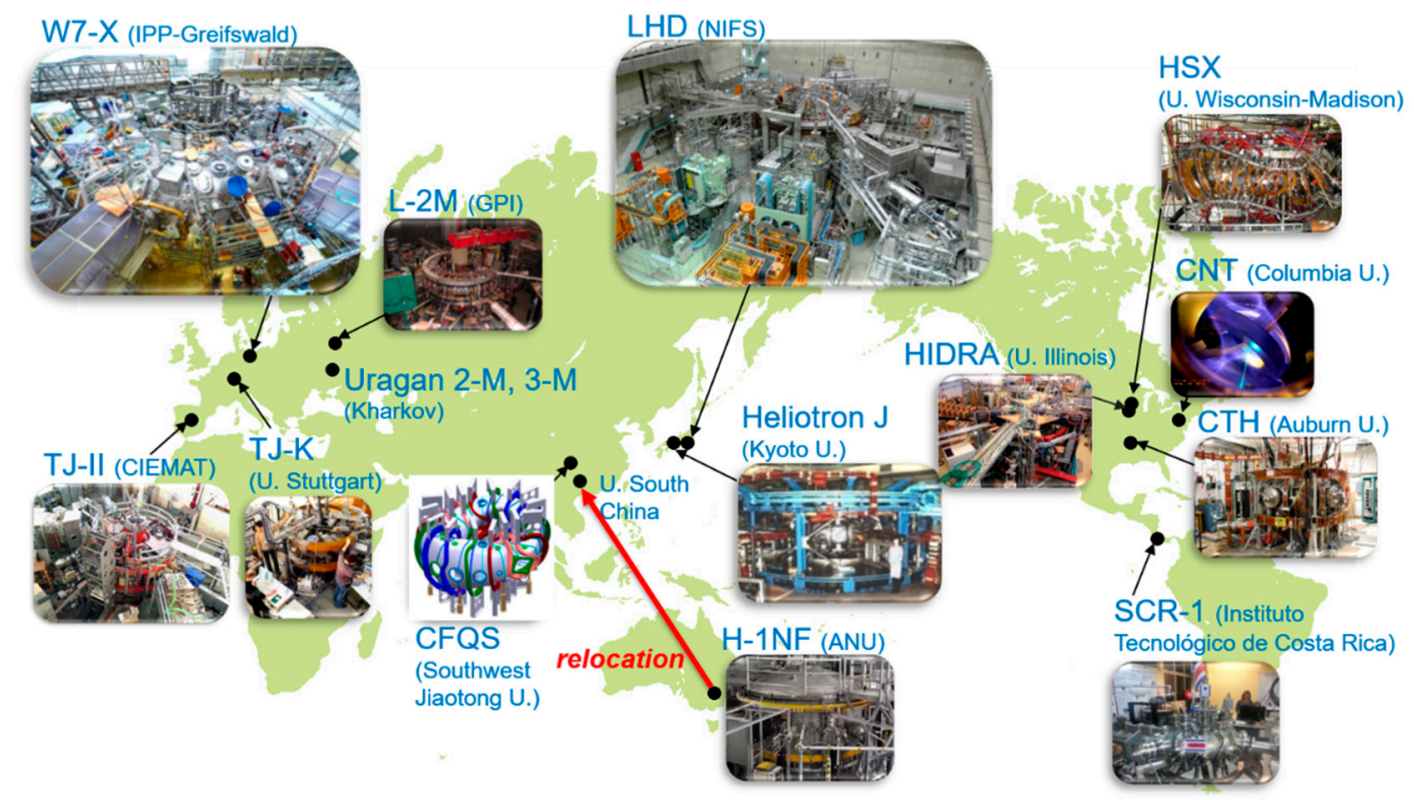

Figure 1. The world-wide helical systems research. This figure has been modified and translated into English from its original version [2].

\section{LHD Project Entering Deuterium Experiment Phase}

Progress in both the physics and engineering aspects of hydrogen experiment phase of LHD has been summarized in recent reviews [6,7]. More specifically, engineering aspects which were the focus of Ref. [6] include the reliable operation of the LHD's large-scale superconducting magnetic system, progress in heating systems, the closed helical diverter, and the successful development and installation of a tritium removal system (installed for use in deuterium experiments). Conversely, in Ref. [7], plasma parameters (e.g., density, temperature, beta values, and long-pulse operation) and their extension in the hydrogen experiment phase were reviewed along with associated physics findings and understandings.

One of the highlights of the hydrogen experiment phase has been the demonstration of a $47 \mathrm{~min}$ 39 s-long discharge, with a few-keV range, achieving the world record in total injected energy (3.36 GJ) [8]. The LHD has explored a world-leading long-pulse operation regime, although its fusion triple product is much lower than those of break-even tokamaks. Complementary research involving helical systems and tokamaks has been envisaged to work towards a steady-state and high-performance fusion reactor regime. Plasma parameters such as temperature, density, and beta value have also steadily developed during the hydrogen experiment phase, as a result of the reliable large-scale superconducting magnet system and physics findings as well as a steady increase in heating power.

Based on this progress, the LHD has entered its deuterium experiment phase (i.e., a more fusion-relevant phase), with the first deuterium plasma on 7 March 2017. The most notable result from the first deuterium campaign was the achievement of an ion temperature of $10 \mathrm{keV}$ [9], as shown in Figure 2. This was a milestone in helical fusion research, in the sense that helical plasma has now reached one of the conditions for fusion. This achievement was made possible by the confinement improvement in deuterium plasmas compared to hydrogen plasmas [9], in addition to an increase in ion heating power via upgraded neutral beam injection (NBI) [10], careful choice of magnetic configuration to retain the heating efficiency of NBI, and the extensive wall conditioning [9]. Experimental observations of the so-called "isotope effect" have taken place not only in high-ion-temperature 
plasmas but also in pure electron cyclotron heated $(\mathrm{ECH})$ plasmas during LHD deuterium experiments. However, these findings need to be investigated further [11-14] to clarify the mechanism of the isotope effect. A simultaneous increase in ion and electron temperatures should be pursued in subsequent deuterium campaigns.

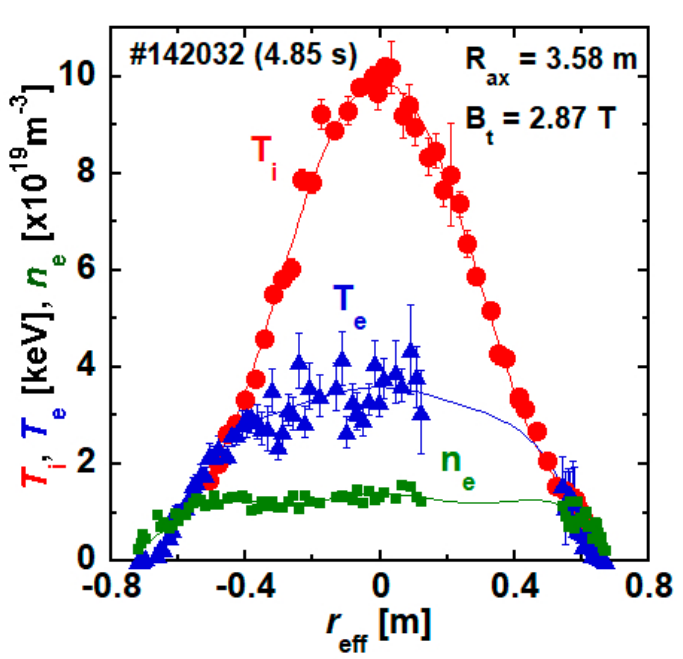

Figure 2. The achievement of an ion temperature of $10 \mathrm{keV}$ in the first deuterium campaign of the Large Helical Device (LHD). The ion temperature profile of $10 \mathrm{keV}$, along with the electron temperature and density profiles, is depicted. The $r_{\text {eff }}$ denotes the effective plasma minor radius (negative values correspond to the inner side of a torus), which is defined as the radius of the equivalent simple torus which encloses the same volume as the flux surface of interest. (This figure is modified from Figure $5 \mathrm{~g}$ in [9]).

Neutrons produced in deuterium plasmas in conjunction with a well-prepared set of neutron diagnostics [15] (e.g., neutron emission rate and triton burn-up ratio) have provided the capacity for the quantitative assessment of the energetic particles' confinement property [16,17] and their interaction with MHD modes [18,19] in LHD plasmas.

The first deuterium campaign has already provided interesting physics findings such as those on impurity behavior [20,21] and the penetration threshold of resonant magnetic perturbation (RMP) [22]. It has also made progress in engineering aspects, including negative-ion-based (NB) injectors [23] and neutron flux distribution in LHD torus hall [24]. All these findings will be presented in other opportunities.

\section{Conceptual Design of the LHD-Type Helical Fusion Reactor FFHR-d1}

Based on progress in both the physics and engineering aspects of the LHD project, conceptual design activity has been extensively conducted for an LHD-type helical fusion reactor-the so-called FFHR-d1 [25]. Its expected fusion power is $3 \mathrm{GW}$. It has the following principal device parameters: major radius of $15.6 \mathrm{~m}$ (four times larger than that of LHD), magnetic field strength of $4.7 \mathrm{~T}$ (at the helical coil center), plasma volume of $1900 \mathrm{~m}^{3}$, and stored magnetic energy of $170 \mathrm{GJ}$. The envisaged plasma parameters are as follows [25]: the central density is $\sim 1.5 \times 10^{19} \mathrm{~m}^{-3}$, the central electron temperature $\sim 16.5 \mathrm{keV}$, and the energy confinement time $\sim 1.5 \mathrm{~s}$. The operation point is explored using a systems code (HELIOSCOPE [26]). An operational point with $Q>10$ has been found with a sub-ignition based on LHD data from hydrogen experiments, where $Q$ is the fusion energy gain factor. Confinement improvement that has been identified in LHD deuterium experiments should widen the scope of the operation. Quantitative assessment for start-up scenarios reaching such an identified operation point has also largely been achieved, as reported in Ref. [27]. Scenario developments have been conducted based on the time evolution of plasma radial profiles by solving 1D transport equations. 
Since this 1D transport code merely employs a simple empirical transport model deduced from LHD experimental results, consistency with detailed physics criteria such as MHD stability and neoclassical transport should be checked by integrating numerical modules for physics analyses [28] that are being or were already validated by LHD experiments. Using these models, control algorithms of auxiliary heating power and fueling amounts have been examined to reach the identified operation point. In this example, smooth control of fusion power was successfully confirmed. In this way, conceptual design of the LHD-type helical fusion reactor FFHR-d1 has progressed, incorporating the time evolution of plasma profiles. A derivation of FFHR-d1, the so-called FFHR-c1, has also been designed with targeting year-long electric power generation by allowing for auxiliary heating along with innovative ideas for its engineering system [29].

\section{LHD as a Platform for Wide-Ranging Plasma Research}

LHD plasmas have been utilized not only for fusion research, but also for wide-ranging plasma research by making use of its steady-state and well diagnosed plasma parameters (e.g., temperature). In this section, two examples of such contributions made by the LHD are introduced.

The first example is the LHD's use in spectroscopic study. Spectroscopic studies have been systematically conducted using LHD on a variety of heavy elements relevant to fusion, as well as in other fields, from basic atomic physics to plasma applications. In the periodic table shown in Figure 3, elements which have been injected into LHD plasmas by means of gas puff or tracer encapsulated solid pellet (TESPEL) [30] are labelled by year. The most frequently studied element is tungsten, which will be used as a material for the ITER diverter. Iron has been investigated for its application in solar astrophysics. Tin, xenon, and lanthanide elements are candidate materials for a light source in EUV lithography. Very heavy elements such as platinum and gold may be used for a water-window light source in biological microscopy.

\begin{tabular}{|c|c|c|c|c|c|c|c|c|c|c|c|c|c|c|c|c|c|}
\hline${ }^{19} \mathrm{~K}$ & ${ }^{20} \mathrm{Ca}$ & ${ }^{21} \mathrm{Sc}$ & ${ }^{22} \mathrm{Ti}$ & $\stackrel{23}{V}$ & ${ }^{24} \mathrm{Cr}$ & $\stackrel{25}{25}$ & $\stackrel{26}{\mathrm{~F} e}$ & ${ }^{27} \mathrm{Co}$ & ${ }^{28} \mathrm{Ni}$ & ${ }^{29} \mathrm{Cu}$ & ${ }^{30} \mathrm{Zn}$ & $\stackrel{31}{\mathrm{Ga}}$ & ${ }^{32} \mathrm{Ge}$ & ${ }^{33} \mathrm{~s}$ & ${ }^{34} \mathrm{Se}$ & ${ }^{35} \mathrm{Br}$ & ${ }^{36} \mathrm{Kr}$ \\
\hline${ }^{37} \mathrm{Rb}$ & ${ }^{38} \mathrm{Sr}$ & $Y$ & ${ }^{40} \mathrm{Zr}$ & $\mathrm{Nb}$ & 10 & ${ }^{43} \mathrm{Tc}$ & Ru & $\stackrel{45}{\mathrm{R} h}$ & $\stackrel{46}{\mathrm{P}} \mathrm{d}$ & $\mathrm{Ag}$ & ${ }^{48} \mathrm{C} d$ & $\stackrel{49}{\mathrm{In}}$ & $\stackrel{50}{5} n$ & ${ }^{51} \mathrm{Sb}$ & $\stackrel{52}{\mathrm{Te}}$ & ${ }^{53} \mathrm{I}$ & $\stackrel{54}{X}^{54}$ \\
\hline Cs & $\stackrel{56}{\mathrm{Ba}}$ & & $\stackrel{72}{\mathrm{Hf}}$ & ${ }^{73} \mathrm{Ta}$ & ${ }^{74} \mathrm{~W}$ & $\stackrel{75}{\operatorname{Re}}$ & ${ }^{76}$ Os & ${ }^{77} \mathrm{Ir}$ & ${ }^{78} \mathrm{Pt}$ & $\stackrel{79}{\mathrm{Au}}$ & $\stackrel{80}{\mathrm{Hg}}$ & ${ }^{81} \mathrm{TI}$ & $\stackrel{82}{\mathrm{~Pb}}$ & ${ }^{83} \mathrm{Bi}$ & $\stackrel{84}{\mathrm{P}}$ & ${ }^{85} \mathrm{At}$ & $\stackrel{86}{R}$ \\
\hline & & & $-a$ & $\mathrm{Ce}$ & $\stackrel{59}{\mathrm{Pr}}$ & $\stackrel{60}{N} d$ & $\stackrel{61}{\mathrm{P}} \mathrm{m}$ & ${ }^{62} \mathrm{~m}$ & $\stackrel{63}{\mathrm{E} u}$ & ${ }^{64} \mathrm{Gd}$ & $\mathrm{Tb}$ & ${ }^{66}{ }^{66}$ & $\stackrel{67}{\mathrm{Ho}}$ & ${ }^{68} \mathrm{Er}$ & T9 & $\stackrel{70}{Y b b}$ & Lu \\
\hline
\end{tabular}

Injected into LHD: 2011, 2012, 2013, 2014, 2017

Figure 3. Elements which have been injected into LHD plasmas are shown in this periodic table, with the year of injection indicated by colors.

With the development of an experimental database for several elements, new spectral lines have been identified for the first time via LHD. By controlling the heating (and thus the electron temperature), the temperature dependence of the EUV spectrum can be systematically obtained. This is the unique advantage of the LHD having excellent spatial and temporal resolutions for electron temperature measurement using a Thomson scattering system. In the case of terbium ions, as described in [30], the spectrum is discrete at electron temperatures above $1 \mathrm{keV}$ and is composed of higher charge states around a $\mathrm{Cu}$-like ion, which has been found for the first time in LHD. As the electron temperature drops below $0.5 \mathrm{keV}$, the spectrum becomes quasi-continuous because the dominant charge states become lower, eventually producing Ag-like ions. Similarly, several isolated spectral lines have been found experimentally for the first time in the LHD, as has been reported in Refs. [31,32]. Z-dependence of the lanthanide spectra has also been studied, and has been recently discussed in Ref. [33].

A second example of the LHD's use in plasma research is the development of a new type of interferometer for electron density measurement. Interferometers are one of the main types of electron density diagnostic systems. However, interferometers suffer from large measurement errors caused by 
mechanical vibrations and changes in air conditions. The installation of vibration isolation systems and the control of air are thus required.

A new type of interferometer called a "dispersion interferometer" is insensitive to mechanical vibrations. It is essentially an interferometer, but it can cancel vibration components automatically by using the second harmonic component $2 \omega$ and a special interferometer configuration, as shown in Figure 4 (all details can be found in Ref. [34]). The second harmonic components are generated twice from the laser fundamental component $\omega$ with nonlinear crystals (i.e., once before and once after the plasma passage) and the interference signal between two second harmonics $I_{\mathrm{DC}}+I_{\mathrm{AC}} \cos \left(1.5 \varphi_{\mathrm{p}}\right)$ is detected, where $I_{\mathrm{DC}}, I_{\mathrm{AC}}$, and $\varphi_{\mathrm{p}}$ denote $\mathrm{DC}$ and $\mathrm{AC}$ values determined by the laser intensity, and the phase shift caused by a plasma, respectively. Since these two wavelength components have almost identical optical paths, the phase shifts caused by vibrations and by the air are the same. By contrast, phase shifts caused by the plasma differ between two wavelength components due to the dispersion of a plasma. Since the phase of the interference signal from which the electron density is calculated is the subtraction of the phases of the two second harmonic components, the phases due to vibrations and air are cancelled and only that due to $\varphi_{\mathrm{p}}$ remains. This is the reason for the invulnerability of the dispersion interferometer to vibrations and air. We have been developing the dispersion interferometer and have implemented phase modulation for further enhancement of resolutions. The interferometer has also been installed in the LHD to demonstrate its feasibility as an electron density diagnostic system for fusion plasmas. Following its successful demonstration within the LHD, it has been decided that it will be installed as a density measurement system for the first plasma of ITER. It is currently being designed and tested for ITER [35].

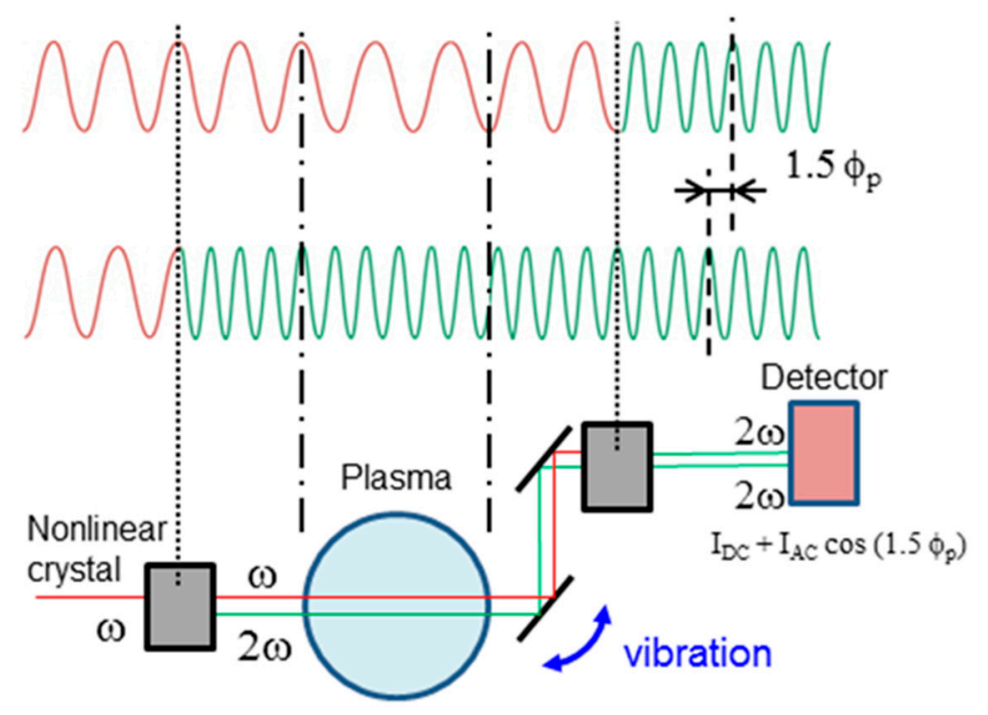

Figure 4. Schematic arrangement of the dispersion interferometer. The dispersion interferometer uses a mixture of the fundamental $\omega$ and second harmonic $2 \omega$ light as a probe beam. The second harmonic light is generated from the incident fundamental light with a nonlinear crystal. After passing through a plasma, the fundamental light is converted to the second harmonic light, and an interference signal between two second harmonic lights is detected. The light path of the second harmonic light generated by a nonlinear crystal is almost the same as that of the fundamental one. Hence, the variations of the light path length caused by mechanical vibrations are the same between the two lights. On the other hand, the phase shifts caused by the plasma are different due to the dispersion of plasma. As a result, phase shifts caused by vibrations are cancelled and only that belonging to the plasma $1.5 \varphi_{\mathrm{p}}$ remains in the interference signal, because the phase of the interference signal is a subtraction of the phases of the two second harmonic lights. In this way, the dispersion interferometer is free from mechanical vibrations. 
Moreover, the dispersion interferometer has been proven to be effective for atmospheric pressure plasmas, not merely for fusion plasmas. Conventional interferometry for atmospheric pressure plasma is not straightforward, because changes in air pressure due to heating by plasma cause a 10-100 times larger phase shift than that caused by a plasma. However, the dispersion interferometer can significantly suppress the effect of air, similarly to how mechanical vibrations do. Proof-of-principle experiments for atmospheric pressure plasmas have been conducted with a dispersion interferometer that was developed for the LHD [36]. As shown in Figure 5, the phase shift quickly increases and decreases when the discharge current turns on and off, respectively. These are the phase shifts caused by the plasma, which corresponds to $1.4 \times 10^{20} \mathrm{~m}^{-3}$. Air also has dispersion, although it is minor. The gradual decrease in the phase shift immediately following the plasma ignition is caused by the dispersion of air. Even though effects due to air remain, the dispersion interferometer enables us to distinguish the plasma phase shift and to evaluate the electron density. In this way, new diagnostics developed within the LHD have been able to contribute not only to fusion plasmas but also to atmospheric pressure plasmas.

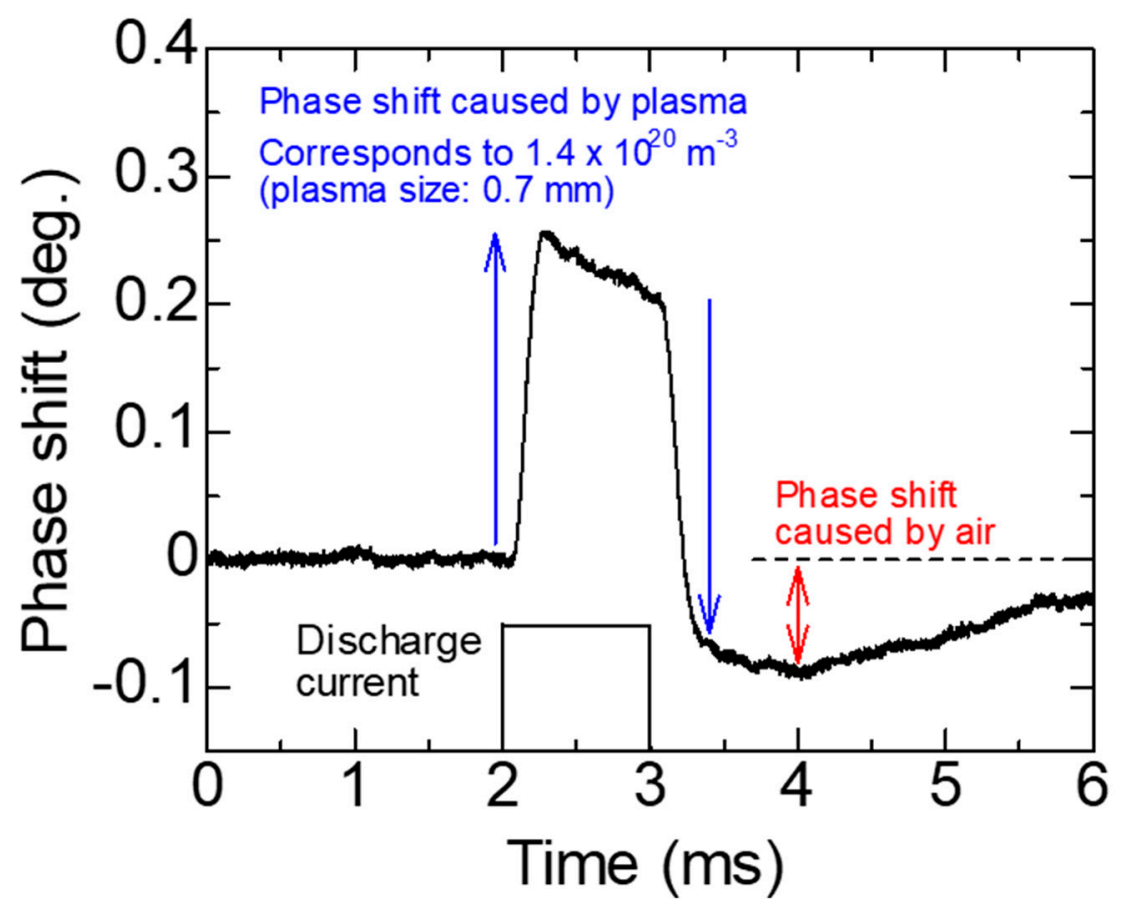

Figure 5. Example of electron density measurements of atmospheric pressure nitrogen plasmas.

\section{Conclusions}

The LHD has progressed as a large-scale superconducting device since 1998, having demonstrated its advantageous capacity for steady-state operation. It has now entered its advanced deuterium experiment phase. A fusion-relevant ion temperature of $10 \mathrm{keV}$ was successfully achieved during the first deuterium campaign. This was a milestone achievement in helical systems research. The LHD will continue to provide research opportunities for reactor-relevant regimes (including high-performance and steady-state plasmas). Ongoing research in the LHD should provide a firm basis for high-precision predictability towards a steady-state helical fusion reactor. The LHD also acts as a platform for diagnostics development and shows promise for applications in wide-ranging plasma research.

Funding: This research was funded by the Ministry of Education, Culture, Sports, Science and Technology (MEXT), Japan.

Acknowledgments: The author would like to acknowledge all domestic and international collaborators for their continued and extensive cooperation. Technical staff are also greatly appreciated for their devoted efforts in 
maintaining and running the LHD. The LHD's experiments and all of NIFS' activities have been continuously and strongly supported by the Ministry of Education, Culture, Sports, Science and Technology (MEXT), Japan.

Conflicts of Interest: The author declares no conflicts of interest.

\section{References}

1. Takeiri, Y.; Morisaki, T.; Osakabe, M.; Yokoyama, M.; Sakakibara, S.; Takahashi, H.; Nakamura, Y.; Oishi, T.; Motojima, G.; Murakami, S.; et al. Extension of the operational regime of the LHD towards a deuterium experiment. Nucl. Fusion 2017, 57, 102023. [CrossRef]

2. Yokoyama, M. History and status of helical fusion research. OHM Magazine, 5 December 2017, pp. 18-23. (In Japanese)

3. Klinger, T.; Alonso, A.; Bozhenkov, S.; Burhenn, R.; Dinklage, A.; Fuchert, G.; Geiger, G.; Grulke, O.; Langenberg, A.; Hirsch, M.; et al. Performance and properties of the first plasmas of Wendelstein 7-X. Plasma Phys. Control. Fusion 2017, 59, 014018. [CrossRef]

4. Vargas, V.I.; Mora, J.; Asenjo, J.; Zamora, E.; Otarola, C.; Barillas, L.; Carvajal-Godínez, J.; González-Gómez, J.; Soto-Soto, C.; Piedras, C. Constructing a small modular stellarator in Latin America. J. Phys. Conf. Ser. 2015, 591, 012016. [CrossRef]

5. Liu, H.; Shimizu, A.; Isobe, M.; Okamura, S.; Nishimura, S.; Suzuki, C.; Xu, Y.; Zhang, X.; Liu, B.; Huang, J.; et al. Magnetic Configuration and Modular Coil Design for the Chinese First Quasi-Axisymmetric Stellarator. Plasma Fusion Res. 2018, 13, 3405067. [CrossRef]

6. Takeiri, Y. Prospect Toward Steady-State Helical Fusion Reactor Based on Progress of LHD Project Entering the Deuterium Experiment Phase. IEEE Trans. Plasma Sci. 2018, 46, 1141-1148. [CrossRef]

7. Takeiri, Y. The Large Helical Device-Entering Deuterium Experiment Phase Toward Steady-State Helical Fusion Reactor Based on Achievements in Hydrogen Experiment Phase. IEEE Trans. Plasma Sci. 2018, 46, 2348-2353. [CrossRef]

8. Seki, T.; Mutoh, T.; Saito, K.; Kasahara, H.; Seki, R.; Kamio, S.; Nomura, G.; Zhao, Y.; Wang, S.; LHD Experiment Group. ICRF Heating Experiment on LHD in Foreseeing a Future Fusion Device. Plasma Fusion Res. 2015, 10, 3405046. [CrossRef]

9. Takahashi, H.; Nagaoka, K.; Murakami, S.; Osakabe, M.; Nakano, H.; Ida, K.; Tsujimura, T.I.; Kubo, S.; Kobayashi, T.; Tanaka, K.; et al. Realization of high Ti plasmas and confinement characteristics of ITB plasmas in the LHD deuterium experiments. Nucl. Fusion 2018, 58, 106028. [CrossRef]

10. Osakabe, M.; Isobe, M.; Tanaka, M.; Motojima, G.; Tsumori, K.; Yokoyama, M.; Morisaki, T.; Takeiri, Y.; LHD Experiment Group. Preparation and Commissioning for the LHD Deuterium Experiment. IEEE Trans. Plasma Sci. 2018, 46, 2324-2331. [CrossRef]

11. Nakata, M.; Nunami, M.; Sugama, H.; Watanabe, T.H. Isotope Effects on Trapped-Electron-Mode Driven Turbulence and Zonal Flows in Helical and Tokamak Plasmas. Phys. Rev. Lett. 2017, 118, 165002. [CrossRef]

12. Warmer, F.; Takahashi, H.; Tanaka, K.; Yoshimura, Y.; Beidler, C.D.; Peterson, B.; Igami, H.; Ido, T.; Seki, R.; Nakata, M.; et al. Energy confinement of hydrogen and deuterium electron-root plasmas in the Large Helical Device. Nucl. Fusion 2018, 58, 106025. [CrossRef]

13. Yamada, H.; Tanaka, K.; Tokuzawa, T.; Seki, R.; Suzuki, C.; Yokoyama, M.; Ida, K.; Yoshimura, M.; Fujii, K.; Yamaguchi, H.; et al. Characterization of Isotope Effect on Confinement of Dimensionally Similar NBI-Heated Plasmas in LHD. Presented at the 27th IAEA Fusion Energy Conference, Gandhinagar, India, 22-27 October 2018; paper EX/P3-5.

14. Tanaka, K.; Nakata, M.; Ohtani, Y.; Tsujimura, T.I.; Takahashi, H.; Yokoyama, M.; Warmer, F.; The LHD Experiment Group. Isotope effects on confinement and turbulence in ECRH plasma of LHD. Presented at the 27th IAEA Fusion Energy Conference, Gandhinagar, India, 22-27 October 2018. paper EX/P3-6.

15. Isobe, M.; Ogawa, K.; Nishitani, T.; Miyake, H.; Kobuchi, T.; Pu, N.; Kawase, H.; Takada, E.; Tanaka, T.; Li, S.; et al. Neutron Diagnostics in the Large Helical Device. IEEE Trans. Plasma Sci. 2018, 46, 2050-2058. [CrossRef]

16. Isobe, M.; Ogawa, K.; Nishitani, T.; Pu, N.; Kawase, H.; Seki, R.; Nuga, H.; Takada, E.; Murakami, S.; Suzuki, Y.; et al. Fusion neutron production with deuterium neutral beam injection and enhancement of energetic-particle physics study in the large helical device. Nucl. Fusion 2018, 58, 082004. [CrossRef] 
17. Ogawa, K.; Isobe, M.; Nishitani, T.; Murakami, S.; Seki, R.; Nuga, H.; Kamio, S.; Fujiwara, Y.; Yamaguchi, H.; Kawase, H.; et al. Energetic-ion Confinement Studies by using Comprehensive Neutron Diagnostics in the Large Helical Device. Presented at the 27th IAEA Fusion Energy Conference, Gandhinagar, India, 22-27 October 2018; paper EX/P3-20.

18. Bando, T.; Ohdachi, S.; Isobe, M.; Suzuki, Y.; Toi, K.; Nagaoka, K.; Takahashi, H.; Seki, R.; Du, X.D.; Ogawa, K.; et al. Excitation of helically-trapped-energetic-ion driven resistive interchange modes with intense deuterium beam injection and enhanced effect on beam ions/bulk plasmas of LHD. Plasma Phys. Control. Fusion 2018, 58, 082025. [CrossRef]

19. Ohdachi, S.; Bando, T.; Nagaoka, K.; Takahashi, H.; Suzuki, Y.; Watanabe, K.Y.; Du, X.D.; Toi, K.; Osakabe, M.; Morisaki, T. Excitation mechanism of the energetic particle driven resistive interchange mode and strategy to control the mode in Large Helical Device. Presented at the 27th IAEA Fusion Energy Conference, Gandhinagar, India, 22-27 October 2018; paper EX/1-3Rb.

20. Ida, K.; Sakamoto, R.; Yoshinuma, M.; Yamazaki, K.; Kobayashi, T.; The LHD Experiment Group. Isotope effect on impurity and bulk ion particle transport in the Large Helical Device. Presented at the 27th IAEA Fusion Energy Conference, Gandhinagar, India, 22-27 October 2018; paper EX/10-1.

21. Oishi, T.; Morita, S.; Kobayashi, M.; Kawamura, G.; Liu, Y.; Goto, M.; The LHD Experiment Group. Effect of deuterium plasmas on carbon impurity transport in the edge stochastic magnetic field layer of Large Helical Device. Presented at the 27th IAEA Fusion Energy Conference, Gandhinagar, India, 22-27 October 2018; paper EX/P3-11.

22. Watanabe, K.Y.; Sakakibara, S.; Narushima, Y.; Ohdachi, S.; Suzuki, Y.; Takemura, Y.; The LHD Experiment Group. Dependence of RMP penetration threshold on plasma parameters and ion species in helical plasmas. Presented at the 27th IAEA Fusion Energy Conference, Gandhinagar, India, 22-27 October 2018. paper EX/P3-15.

23. Ikeda, K. Exploring Deuterium Beam Operation and Behavior of Co-Extracted Electron in Negative-Ion-Based Neutral Beam Injector. Presented at the 27th IAEA Fusion Energy Conference, Gandhinagar, India, 22-27 October 2018; paper FIP/P1-54.

24. Kobayashi, M.; Tanaka, T.; Nishitani, T.; Ogawa, K.; Isobe, M.; Motojima, G.; Kato, A.; Saze, T.; Yoshihashi, S.; Osakabe, M.; The LHD Experiment Group. Neutron flux distributions in the LHD torus hall evaluated by an imaging plate technique in the first campaign of deuterium plasma experiment. Presented at the 27 th IAEA Fusion Energy Conference, Gandhinagar, India, 22-27 October 2018; paper FIP/P3-4.

25. Sagara, A.; Miyazawa, J.; Tamura, H.; Tanaka, T.; Goto, T.; Yanagi, N.; Sakamoto, R.; Masuzaki, S.; Ohtani, H.; The FFHR Design Group. Two conceptual designs of helical fusion reactor FFHR-d1A based on ITER technologies and challenging ideas. Nucl. Fusion 2017, 086046. [CrossRef]

26. Goto, T.; Suzuki, Y.; Yanagi, N.; Watanabe, K.Y.; Imagawa, S.; Sagara, A. Importance of helical pitch parameter in LHD-type heliotron reactor designs. Nucl. Fusion 2011, 51, 083045. [CrossRef]

27. Goto, T.; Miyazawa, J.; Sakamoto, R.; Suzuki, Y.; Suzuki, C.; Seki, R.; Satake, S.; Huang, B.; Nunami, M.; Yokoyama, M.; et al. Development of a real-time simulation tool towards self-consistent scenario of plasma start-up and sustainment on helical fusion reactor FFHR-d1. Nucl. Fusion 2017, 57, 066011. [CrossRef]

28. Yokoyama, M.; Seki, R.; Suzuki, C.; Sato, M.; Emoto, M.; Murakami, S.; Osakabe, M.; Tsujimura, T.I.; Yoshimura, Y.; Ido, T.; et al. Extended capability of the integrated transport analysis suite, TASK3D-a, for LHD experiment. Nucl. Fusion 2017, 57, 126016. [CrossRef]

29. Miyazawa, J.; Goto, T.; Tamura, H.; Tanaka, T.; Yanagi, N.; Murase, T.; Sakamoto, R.; Masuzaki, S.; Ohgo, T.; Sagara, A.; et al. Maintainability of the helical reactor FFHR-c1 equipped with the liquid metal divertor and cartridge-type blankets. Fusion Eng. Des. 2018, 136 Pt B, 1278-1285. [CrossRef]

30. Sudo, S.; Tamura, N. Tracer-encapsulated solid pellet injection system. Rev. Sci. Instrum. 2012, 83, 023503. [CrossRef]

31. Suzuki, C.; Koike, F.; Murakami, I.; Tamura, N.; Sudo, S. Temperature dependent EUV spectra of Gd, Tb and Dy ions observed in the Large Helical Device. J. Phys. B At. Mol. Opt. Phys. 2015, 48, 144012. [CrossRef]

32. Suzuki, C.; Murakami, I.; Koike, F.; Tamura, N.; Sakaue, H.A.; Morita, S.; Goto, M.; Kato, D.; Ohashi, H.; Higashiguchi, T.; et al. Extreme ultraviolet spectroscopy and atomic models of highly charged heavy ions in the Large Helical Device. Plasma Phys. Control. Fusion 2017, 59, 014009. [CrossRef]

33. Suzuki, C.; Koike, F.; Murakami, I.; Tamura, N.; Sudo, S. Systematic Observation of EUV Spectra from Highly Charged Lanthanide Ions in the Large Helical Device. Atoms 2018, 6, 24. [CrossRef] 
34. Akiyama, T.; Yasuhara, R.; Kawahata, K.; Okajima, S.; Nakayama, K. Dispersion interferometer using modulation amplitudes on LHD. Rev. Sci. Instrum. 2014, 85, 11D301. [CrossRef] [PubMed]

35. Akiyama, T.; Sirinelli, A.; Watts, C.; Shigin, P.; Vayakls, G.; Walsh, M. Design of a dispersion interferometer combined with a polarimeter to increase the electron density measurement reliability on ITER. Rev. Sci. Instrum. 2016, 87, 11E133. [CrossRef] [PubMed]

36. Urabe, K.; Akiyama, T.; Terashima, K. Application of phase-modulated dispersion interferometry to electron-density diagnostics of high-pressure plasma. J. Phys. D Appl. Phys. 2014, 47, 262001. [CrossRef]

(C) 2018 by the author. Licensee MDPI, Basel, Switzerland. This article is an open access article distributed under the terms and conditions of the Creative Commons Attribution (CC BY) license (http:/ / creativecommons.org/licenses/by/4.0/). 